\title{
Article
}

\section{Ab Initio Simulation of the IR Spectrum of Hydrated Kaolinite}

\author{
Victor Yavna*(D), Tatiana Nazdracheva, Andrey Morozov, Yakov Ermolov and Andrei Kochur (D) \\ Department of Physics, Rostov State Transport University, 2 Narodnogo Opolcheniya Sq., \\ 344038 Rostov-on-Don, Russia; nazdracheva98@mail.ru (T.N.); nata-moroz@mail.ru (A.M.); \\ iashaermolov@yandex.ru (Y.E.); agk@rgups.ru (A.K.) \\ * Correspondence: vay@rgups.ru
}

Citation: Yavna, V.; Nazdracheva, T.; Morozov, A.; Ermolov, Y.; Kochur, A. $\mathrm{Ab}$ Initio Simulation of the IR Spectrum of Hydrated Kaolinite. Crystals 2021, 11, 1146. https:// doi.org/10.3390/cryst11091146

Academic Editor: Vladimir Fedin

Received: 22 August 2021

Accepted: 18 September 2021

Published: 20 September 2021

Publisher's Note: MDPI stays neutral with regard to jurisdictional claims in published maps and institutional affiliations.

Copyright: (c) 2021 by the authors. Licensee MDPI, Basel, Switzerland. This article is an open access article distributed under the terms and conditions of the Creative Commons Attribution (CC BY) license (https:// creativecommons.org/licenses/by/ $4.0 /)$.
Abstract: The hydration of the basal surfaces of kaolinite is studied by theoretical methods. The cluster method was used to simulate the positions of atoms. The positions of the atoms of the basal surfaces of dry and hydrated minerals are optimized by minimizing the total energy in the Hartree-Fock approximation. The adsorption energies of water molecules were calculated taking into account the fourth-order correlation corrections of Møller-Plesset perturbation theory. The formation of the IR spectrum of kaolinite in the range of wave numbers $2500-4500 \mathrm{~cm}^{-1}$ is studied. The experimentally observed effect of the change in relative intensity and position of the band with a change in the moisture content of the sample is interpreted.

Keywords: kaolinite; hydration; basal surfaces; cluster method; electronic and spatial structures; Hartree-Fock method; correlation energy

\section{Introduction}

Hydration of clay minerals is currently being studied by experimental and theoretical methods. Interest in such research is associated with a wide range of applications of the information obtained, e.g., in the problems of construction, creation of ceramic products, and solution of environmental problems.

The peculiarities of the hydration process depend on the properties of clay materials [1] and determines the possibility of their use as building materials in the construction of subgrades of highways and railways, and other soil structures. Strength and other geotechnical properties of soil depend both on the history of the hydration process and on its current state [2]. Another problem associated with the peculiarities of the hydration of clay soils used in construction is associated with the possibility of swelling which leads to significant material costs during the operation of artificial earth structures [3]. The use of clay soils in the manufacturing of ceramic products for a wide range of applications requires knowledge of all their properties, including the features of hydration [4]. The importance of studying the adsorption of water by clay particles in environmental problems is evident from the fact that in the Earth's atmosphere, aerosols of mineral dust account for up to $45 \%$ of the total aerosol load of the atmosphere [5].

Experimental methods for studying the process of hydration of clay minerals are based on studies of mechanical properties of moist minerals, as well as on studying their interaction with electromagnetic fields in the microwave and infrared frequency ranges. The use of experimental microwave measurement methods makes it possible to determine real and imaginary parts of the dielectric constant depending on the moisture content of the sample [6].

The method of the IR spectroscopy makes it possible to study not only the electronic and spatial structure of hydrated minerals, but also to perform a quantitative analysis of the mineralogical composition of soils $[7,8]$. The results of studying the IR spectra of clay soils make it possible to calculate a wide range of mechanical properties, including plastic properties and characteristics of shrinkage and tension [9]. 
Among the theoretical methods for calculating the interaction of individual $\mathrm{H}_{2} \mathrm{O}$ molecules and clay particles, the density functional theory (DFT) methods are commonly used. Simulating the hydration of clay particles with basal surfaces of imperfect shape and in conditions of high moisture is usually carried out using molecular dynamics (MD) methods. In [10], the mechanisms of hydration of kaolinite are studied by the DFT methods. Simulations showed that the $\mathrm{H}_{2} \mathrm{O}$ molecules are predominantly adsorbed on the hydroxyl surface of kaolinite. The most energetically favorable position of the water molecule is an active center formed by three hydrogen atoms. The adsorption energy is $0.37 \mathrm{eV}$. On a siloxane surface, which is less hydrophilic, a chemical bond is formed between the hydrogen atoms of the water molecule and two surface oxygen atoms. Calculated adsorption energy was $0.07 \mathrm{eV}$.

In [11], the DFT method was also used to study the interaction between water molecules and the basal surfaces of kaolinite. Simulations at low moisture contents yielded the adsorption energy of $0.57 \mathrm{eV}$ for the hydroxyl surface. In [12], hydration of kaolinite was studied by both DFT and MD methods. It was found that the adsorption energy of one water molecule in different initial positions on the hydroxyl surface of kaolinite is from 72.12 to $19.23 \mathrm{~kJ} / \mathrm{mol}(0.74-0.20 \mathrm{eV})$ or, on average, $0.47 \mathrm{eV}$ per molecule; this is energetically more favorable than on the siloxane surface of kaolinite where the adsorption energy varies from 19.23 to $5.77 \mathrm{~kJ} / \mathrm{mol}(0.20-0.06 \mathrm{eV})$ or, on average, $0.13 \mathrm{eV}$ per molecule. In [13], hydrophilicity of hydroxyl and hydrophobicity of siloxane surfaces of kaolinite were demonstrated by the MD methods.

Theoretical values of the absorption energies reported in the literature, and the conclusions about the properties of surfaces, are in qualitative agreement. The observed scatter of theoretical absorption energies can be explained by the difference in the approximations and models used; this increases the relevance of experimental verification of the results.

In this work, the features of hydration of kaolinite are studied by theoretical methods, which made it possible to understand experimentally recorded changes in the position and intensity of a broad band at $3400 \mathrm{~cm}^{-1}$ in the IR spectrum [14]. It is known that dangling chemical bonds on the lateral surfaces of kaolinite particles take part in the chemical adsorption of both ions and water molecules. This chemical interaction mechanism is responsible for some unique properties of kaolinite, such as amphotericity [15]. In this work, only the hydration of basal surfaces is considered since their area significantly exceeds the area of the lateral surface, and then basal-surfaces processes should be the principal factors determining the optical properties of the samples under study.

\section{Theory and Methods}

\subsection{Cluster Models of Basal Surfaces}

Theoretical studies carried out in this work include the calculation of the electronic states energy and spatial positions of atoms of water and mineral molecules; a cluster model of basal surfaces is used.

The initial construction of the crystal structure of kaolinite was carried out using the experimental data presented at http:/ / rruff.geo.arizona.edu/AMS/amcsd.php, accessed on 22 July 2021 (American Mineralogist Crystal Structure Database, database_code_amcsd 0012237) [16].

A fragment of the crystal structure of the kaolinite layer is shown in Figure 1. Visualization of the mineral structure was performed using the MacMolPlt v 7.7 software package [17]. Here and below, the following designations of atoms are used: $\bullet-\mathrm{Si}, \bullet-\mathrm{Al}$, $\bullet-\mathrm{O}, \bullet-\mathrm{H}$.

When constructing computer models of the basal surfaces, the crystal structure of kaolinite is represented by two single-layer clusters. The siloxane surface cluster contained silicon atoms and surrounding oxygen tetrahedra, and the hydroxyl surface cluster contained aluminum atoms, fragments of oxygen octahedra, and surface hydrogen atoms. Basal surfaces clusters are shown in Figure 2. 


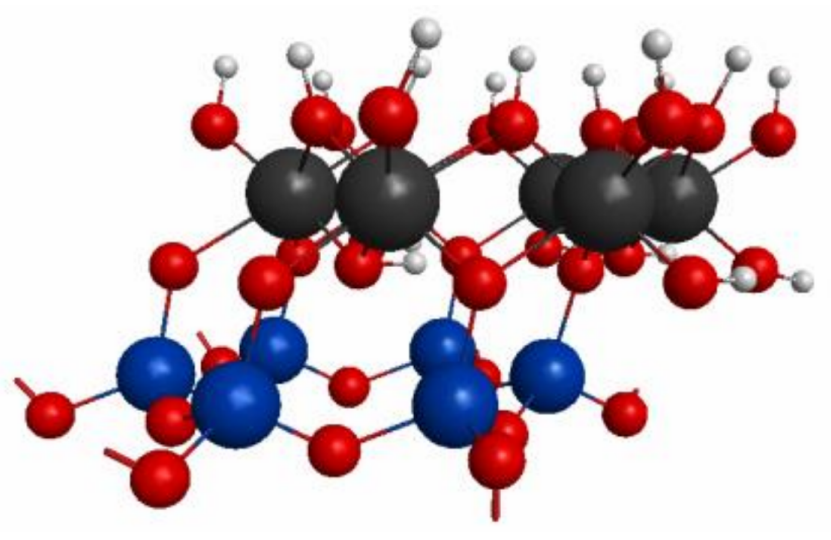

Figure 1. A fragment of the crystal structure of the kaolinite layer.
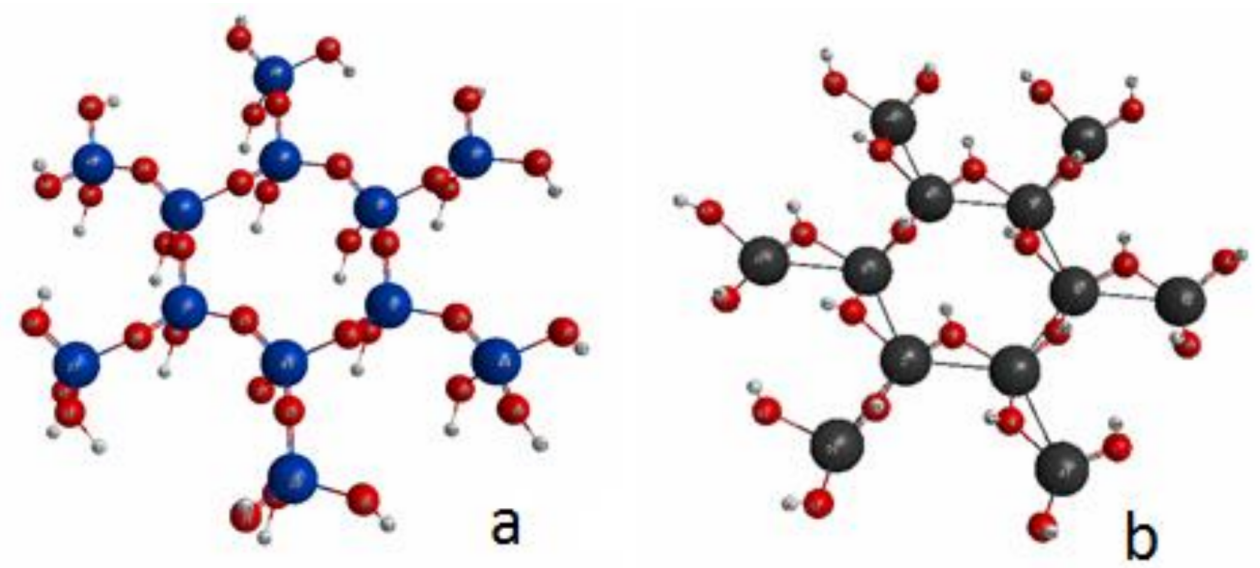

Figure 2. Atomic clusters representing the basal siloxane (a) and hydroxyl (b) surfaces of kaolinite. The atoms are marked in the same way as in Figure 1.

The chemical bonds of atoms that were cut off when constructing the clusters were closed by hydrogen atoms; their vibrations were subsequently disregarded when calculating the IR spectrum. The moistening of kaolinite was simulated by adding water molecules to the clusters. They were placed near the basal surfaces within the central hexagon at a distance from the surface not exceeding $10 \AA$ [12].

When performing the calculations, the position of atoms of $\mathrm{H}_{2} \mathrm{O}$ molecules, the positions of six silicon atoms and six oxygen atoms forming the atomic structure of the hexagon of the siloxane surface (Figure 2a), and the positions of six hydrogen atoms and six oxygen atoms forming the atomic structure of the hexagon of the hydroxyl surface (Figure $2 b$ ) were optimized. The positions of other atoms of the mineral were taken unchanged and coinciding with the experimental ones (Figure 1).

\subsection{Calculation of the Electronic Structure and Energy of Surface Clusters}

Calculations of cluster properties were performed using Firefly v.8.2.0 package [18], which is partially based on the GAMESS (US) source code [19]. Calculations of the optimal position of atoms of basal surfaces clusters were performed in Hartree-Fock approximation. To simulate the wave functions of atoms, we used the N21 basis supplemented for hydrogen and oxygen atoms of surface clusters with optimized coordinates, and the atoms of water molecules, with $\mathrm{p}$ - and d-diffuse functions, respectively. Calculated coordinates and electronic properties of the surface clusters and water were used to calculate the IR spectra.

To refine the Hartree-Fock (HF) energies of the moistened basal surfaces, a calculation was performed within Møller-Plesset perturbation theory taking into account fourth-order 
correlations (MP4). In this case, previously calculated coordinates were used. To simulate the one-electron functions of atoms when calculating the energies, a Slater-type-orbital basis with six Gaussian functions was used.

The use of a limited basis set leads to errors in the calculation of energies commonly referred to as basis set superposition errors (BSSE). The compensation of errors in the energy of interaction of the basal surfaces and the $\mathrm{H}_{2} \mathrm{O}$ molecules clusters was performed by the counterpoise correction scheme (CP) by Boys-Bernardi method [20].

\section{Calculation Results and Discussion}

\subsection{Electronic Structure of Basal Surfaces Clusters}

To assess the quality of the cluster simulation of kaolinite crystal surfaces, the densities of occupied and free electronic states are calculated in this section. For this purpose, calculated one-electron orbital energies of clusters representing siloxane and hydroxyl surfaces were used. According to Section 2.1, when the clusters were formed, to simulate the bonds between them, the dangling chemical bonds were closed with hydrogen atoms. Therefore, when calculating the densities of electronic states from a set of free and occupied one-electron orbitals of the clusters, the states of these hydrogen atoms were removed. The remaining discrete one-electron states of the clusters of the siloxane and hydroxyl basal surfaces were replaced by Lorentzian components with a half-maximum width of $0.02 \mathrm{eV}$. The densities of states are obtained by summing all one-electron components of both clusters; they are presented in Figure 3. The figure also shows the densities of states calculated using the results of the DFT calculation for an infinite crystal performed in [21].

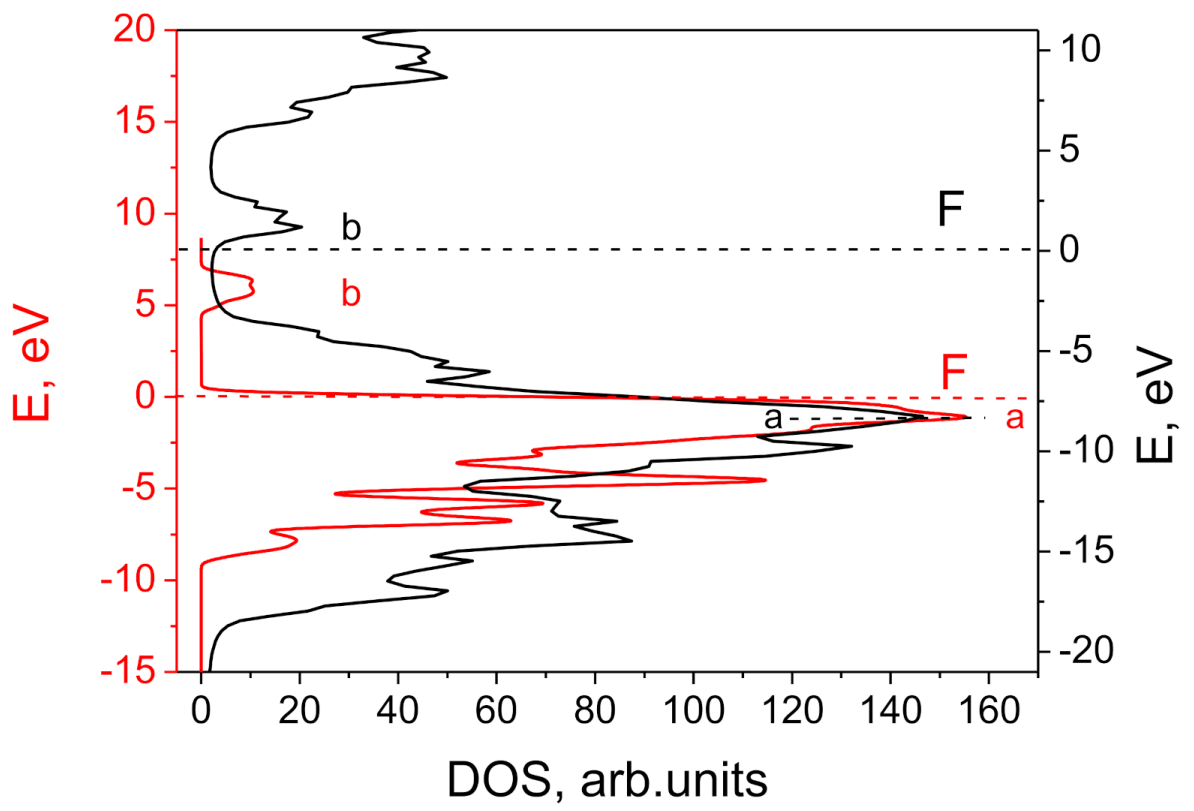

Figure 3. Density of electronic states calculated in this work (black line) and according to the data of [21] (red line). The positions of the density of states maxima of the valence band (a) and the band of free states (b), as well as the position of the Fermi levels (F), are marked.

In Figure 3, the bands of electronic states calculated in this work are shifted by $9.0 \mathrm{eV}$ so that the maxima of the valence band density calculated by the two methods had the same position in the energy scale (position a). Figure 3 shows that such an energy shift leads to a shift of the energy zeros (Fermi levels) in the two calculation methods, by $7.5 \mathrm{eV}$. A shift of energy scales can be explained by the fact that the cluster calculation does not take into account the Madelung potential created by the ions of an infinite crystal.

Comparison of the band shapes calculated in the two considered approaches (Figure 3) allows one to note that, in the cluster approach, the valence band width is larger; however, 
the forbidden gap is reproduced well. In addition, the analysis of the results of this study makes it possible to associate the band of free states (position b) with the 3s states of aluminum, which correlates with the results of [21], where the partial density of the s-type states are 1.5 times larger than that of the p-type states.

\subsection{Sample Moisture}

Studying the effect of moisture on the electronic and spatial structure of the mineral using the cluster model involves performing calculations which take into account a given number of $\mathrm{H}_{2} \mathrm{O}$ molecules. To compare the theoretical results obtained in this case with the experiment, it is necessary to convert the number of adsorbed water molecules into the moisture content of the sample as used in the experimental studies. In this work, this transformation is performed by taking into account the following considerations.

The chemical formula of the unit cell of kaolinite is $\mathrm{Al}_{2} \mathrm{Si}_{2} \mathrm{O}_{5}(\mathrm{OH})_{4}$, and its mass number is 256. To form hexagons of silicon and aluminum (see Figure 1), the atoms of three unit cells are required. In this case, six hexagons of the environment will be formed by one third. Thus, three-unit cells give the total number of hexagons equal to three. Then the mass number of one group of hexagons is $M=256$. A water molecule has a mass number $m_{\mathrm{H} 2 \mathrm{O}}=18$. Then the addition of one water molecule is equivalent to the moisture content of the sample:

$$
w=\frac{m_{H 2 O}}{M} \times 100 \%=7 \%
$$

In the case when the optimization of the coordinates of water molecules leads to their belonging to several hexagons, the moisture value must be adjusted accordingly. Let us illustrate the correction procedure by an example of calculating the moisture content when placing five $\mathrm{H}_{2} \mathrm{O}$ molecules on the siloxane surface of the cluster (Figure 4). We will assume that the molecule placed above the hexagon of the silicon atoms, after optimization of cluster geometry, contributes to the moisture content with a weight of 1 (green rectangle in Figure 4)). If the $\mathrm{H}_{2} \mathrm{O}$ molecule is placed over the side of the hexagon, its weight will be 0.5 , and if it is placed over the vertex of the hexagon, 0.3 (black rectangles in Figure 4). As a result, the total contribution of five $\mathrm{H}_{2} \mathrm{O}$ molecules is 2.3. In this case, the moisture content of the sample is equal to $w=16.3 \%$.

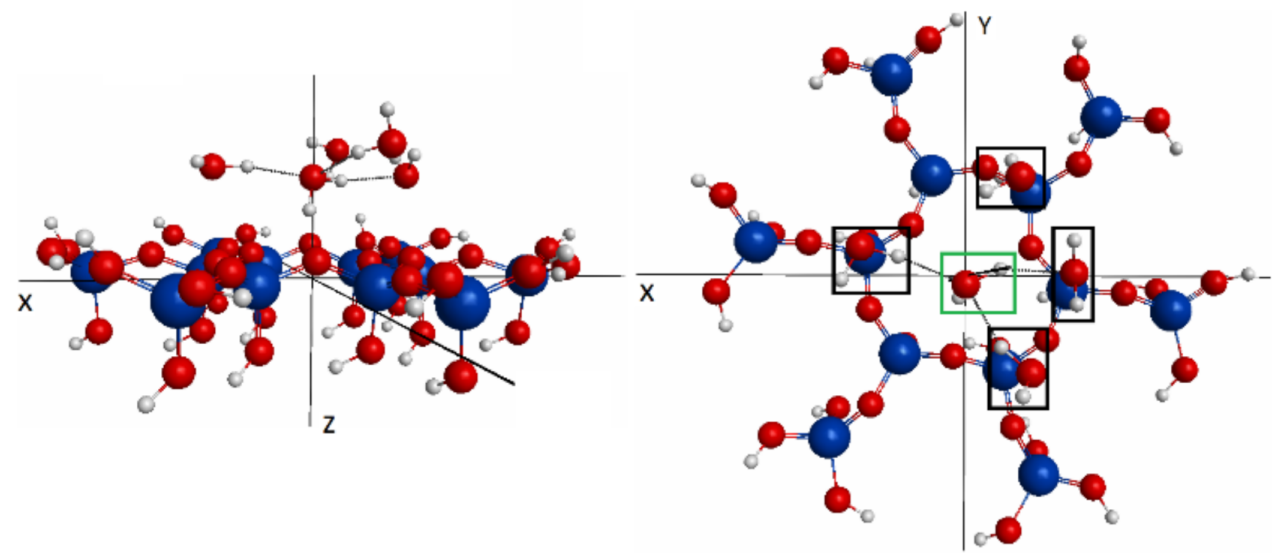

Figure 4. Siloxane surface cluster and five $\mathrm{H}_{2} \mathrm{O}$ molecules.

Table 1 shows the results of applying this procedure to the results of calculations performed in this work. 
Table 1. Number of water molecules included in the calculations and the moisture content of kaolinite samples.

\begin{tabular}{cccccc}
\hline & Siloxane Surface & & \multicolumn{3}{c}{ Hydroxyl Surface } \\
\hline $\begin{array}{c}\text { Number of } \\
\text { Molecules }\end{array}$ & Weight & $\begin{array}{c}\text { Moisure } \\
\text { Content, } \%\end{array}$ & $\begin{array}{c}\text { Number of } \\
\text { Molecules }\end{array}$ & Weight & $\begin{array}{c}\text { Moisure } \\
\text { Content, \% }\end{array}$ \\
\hline 1 & 1 & 7 & 1 & 1 & 7 \\
\hline 2 & 1.3 & 9.3 & 2 & 2 & 14 \\
\hline 3 & 1.7 & 11.7 & 3 & 2.5 & 17.5 \\
\hline 4 & 2 & 14 & 4 & 2.8 & 19.8 \\
\hline 5 & 2.3 & 16.3 & 5 & 3.8 & 26.8 \\
\hline 6 & 2.7 & 18.7 & 6 & 4 & 28 \\
\hline
\end{tabular}

\subsection{Effect of Adsorption on the Geometry of the $\mathrm{H}_{2} \mathrm{O}$ Molecule}

When a water molecule is adsorbed, its atoms are affected both by the atoms of the basal surface of the mineral and the atoms of other water molecules. These interactions will change the geometric characteristics, i.e., the length of the $\mathrm{O}-\mathrm{H}$ bond and the bond angle of the molecule. In [22], it was obtained by the DFT method that at low moisture contents, when all water molecules are near the basal surfaces of kaolinite, the $\mathrm{O}-\mathrm{H}$ distance in water molecules changes insignificantly on average, while the bond angle, depending on the moisture, can vary within the range of $102.1^{\circ}-105.4^{\circ}$. Thus, the change in the average bond angle of the $\mathrm{H}_{2} \mathrm{O}$ molecules being taken into account in the calculations can serve as a measure of the sorption capacity of surfaces varying with moisture.

In this study, it was found that the $\mathrm{O}-\mathrm{H}$ distance of a free molecule equal to $0.95 \AA$, remains unchanged in the entire range of the considered moistures, while the change in the bond angle, equal to $103.93^{\circ}$ for a free molecule, can noticeably change depending on the moisture content and the properties of the sorbing surface. Calculated changes in the average bond angle relative to the angle in a free molecule are shown in Figure 5.

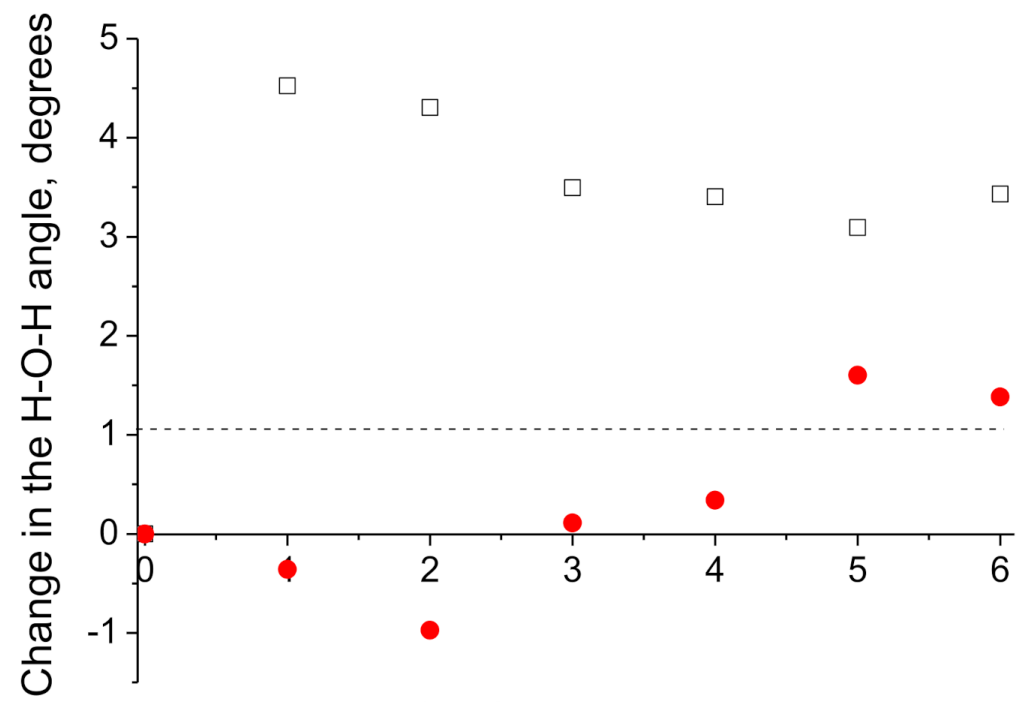

Number of $\mathrm{H}_{2} \mathrm{O}$ molecules per cluster

Figure 5. Average change in the bond angle of the $\mathrm{H}_{2} \mathrm{O}$ molecule depending on the number of water molecules per cluster, and on the type of basal surface ( $\square$-hydroxyl surface, $\bullet$ - siloxane surface). The dashed line shows the change of the average bond angle $\left(1.04^{\circ}\right)$ upon going from a free $\mathrm{H}_{2} \mathrm{O}$ molecule to a water cluster consisting of 6 molecules.

Figure 5 shows that as the number of adsorbed water molecules on the siloxane surface 
increases, the bond angle becomes larger than the initial one, since the role of interaction between water molecules increases on the background of weak interaction with surface ions. When six $\mathrm{H}_{2} \mathrm{O}$ molecules are taken into account in the calculation, the averaged value of the bond angle becomes close to that in the water cluster. Changes in the bond angle, upon going from a free molecule to a cluster of six water molecules, are shown in the figure by the dashed line. The results obtained indicate that the hydrophilicity of the siloxane surface is small.

Upon the adsorption of one $\mathrm{H}_{2} \mathrm{O}$ molecule by the hydroxyl surface of kaolinite, a sharp increase in the bond angle is observed. A further increase in the number of $\mathrm{H}_{2} \mathrm{O}$ molecules placed on the surface leads to a decrease in the interactions stretching the angle. However, when six $\mathrm{H}_{2} \mathrm{O}$ molecules are taken into account, the interaction with the cluster surface is still significant, since the bond angle exceeds by about $2^{\circ}$ that of the bond angle in a free cluster of six water molecules. The results obtained indicate that the hydrophilicity of the hydroxyl surface exceeds that of the siloxane surface; it does not disappear even at a moisture content at the plastic limit, i.e., 25.9\% [23] (Table 1).

In general, the changes in the bond angle of a water molecule depend on moisture content and vary in the range of $103.0^{\circ}-108.5^{\circ}$; this correlates with the data of [22]. An increase in the $\mathrm{H}-\mathrm{O}-\mathrm{H}$ bond angle upon adsorption of water molecules indicates a decrease in the role of interatomic interaction forces within water molecules against the background of their additional interaction with the atoms of the mineral surface, and the atoms of other $\mathrm{H}_{2} \mathrm{O}$ molecules.

\subsection{Adsorption Energy}

Investigations aimed at studying the adsorption of water molecules were carried out by the methods of DFT [10,12], and MD [12,13]. A comparison of the results obtained was made in [14] where it was noted that in the models used, $\mathrm{H}_{2} \mathrm{O}$ molecules are predominantly adsorbed on the hydroxyl surface of kaolinite. The adsorption energies calculated in [10-12] differ insignificantly. The difference may be due to the details of the simulations and the accuracy of the potentials used in the calculations. A review of the results made in [14] makes it possible to note that, in the absence of additional ions, the average adsorption energies on hydroxyl and siloxane basal surfaces in kaolinite differ by a factor of about 5 and amount to 0.5 and $0.1 \mathrm{eV}$, respectively.

In this study, the calculations of the adsorption energy per one water molecule were performed for different numbers of the adsorbed water molecules. To this end, geometry optimization and the calculation of total energies were performed for the following systems:

- $\quad$ Free clusters of basal surfaces $\left(E_{c 0}\right)$;

- Klusters and $n \mathrm{H}_{2} \mathrm{O}$ molecules adsorbed by the basal surfaces $\left(E_{c n \mathrm{H}_{2} \mathrm{O}}\right)$;

- $\quad$ One free $\mathrm{H}_{2} \mathrm{O}$ molecule $\left(E_{\mathrm{H}_{2} \mathrm{O}}\right)$;

- $\quad n$ isolated water molecules with atoms in coordinates optimized in the 'cluster $+n \mathrm{H}_{2} \mathrm{O}^{\prime}$ system $\left(E_{n \mathrm{H}_{2} \mathrm{O}}\right)$.

Now the adsorption energies per one water molecule are determined by the formula:

$$
E_{a d s}=\left(E_{c 0}+n E_{\mathrm{H}_{2} \mathrm{O}}-E_{c n \mathrm{H}_{2} \mathrm{O}}\right) / n
$$

The adsorption energy (2) can be split into separate contributions from water molecules, and from the cluster of basal surfaces. The contribution from water molecules is calculated by

$$
E_{\text {ads }}\left(\mathrm{H}_{2} \mathrm{O}\right)=\left(n E_{\mathrm{H}_{2} \mathrm{O}}-E_{n \mathrm{H}_{2} \mathrm{O}}\right) / n
$$

Contribution from the clusters of basal surfaces is then

$$
E_{a d s}(c)=E_{a d s}-E_{a d s}\left(H_{2} \mathrm{O}\right)
$$


The results of the HF calculations in MP4 approximation are presented in Figure 6, which shows the energies of adsorption on siloxane and hydroxyl surfaces, as well as individual contributions to these values from the surfaces of the mineral.

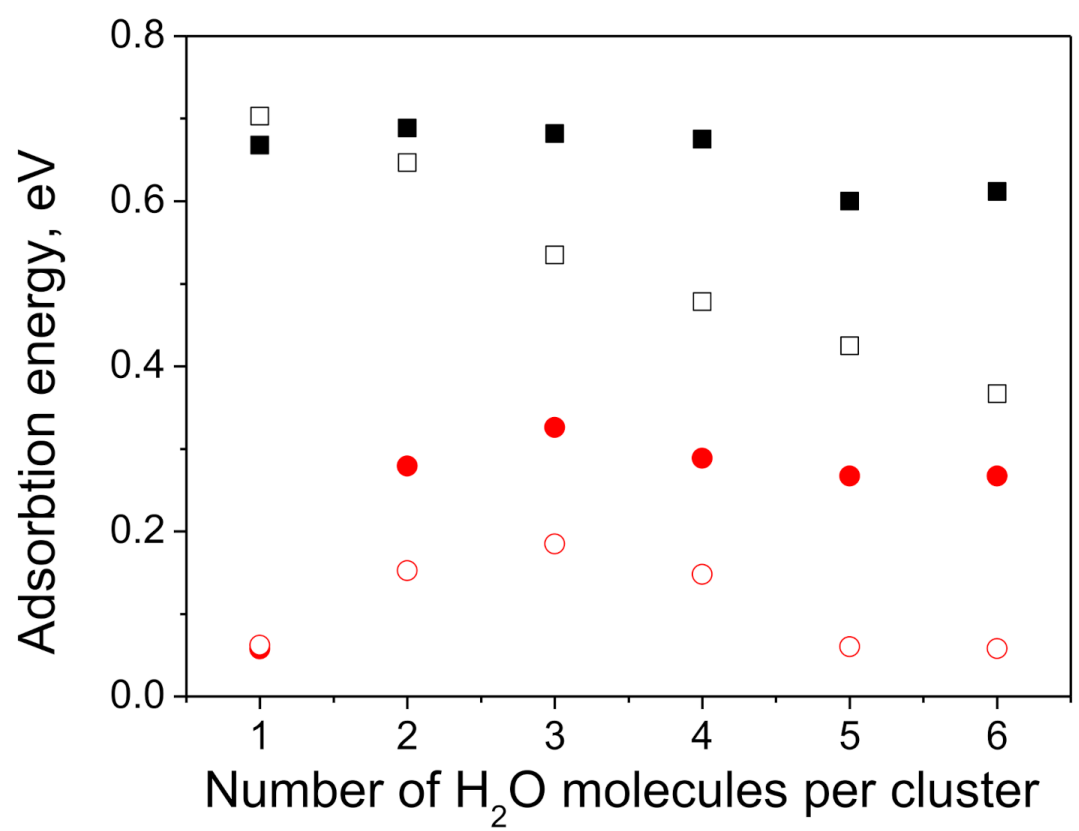

Figure 6. Energies of adsorption of water molecules sorbed by the basal surfaces of kaolinite per molecule. The following designations are used: cluster of the hydroxyl surface and water molecules口; hydroxyl surface cluster- $\square$; cluster of siloxane surface and water molecules-•; siloxane surface cluster- $\bigcirc$.

Figure 6 shows that the hydrophilic nature of the hydroxyl surface can be characterized by the adsorption energy of $0.65 \mathrm{eV}$ for the numbers of the adsorbed molecules from 1 to 6 , i.e., in a wide range of moistures. At the same time, the contribution of the surface to this value decreases with increasing moisture; this change is compensated by an increase of the binding energy within the subsystem of water molecules.

When water molecules are adsorbed by the siloxane surface, hydrophilicity is also observed, but the adsorption energy is noticeably smaller. When one water molecule is adsorbed, the adsorption energy is close to $0.05 \mathrm{eV}$. With an increase in the number of the adsorbed molecules, it increases to $0.3 \mathrm{eV}$; in this case, it is due mostly to the interaction of water molecules with each other.

The BSSE-correction of Formula (2) for calculating the energy of water adsorption on the clusters of basal surfaces was performed according to the Boys-Bernardi approach:

$$
E_{a d s}^{\mathrm{CP}}=E_{a d s}+\delta^{\mathrm{BSSE}}
$$

where the counterpoise correction is designated $\delta^{\mathrm{BSSE}}$ and depends on the optimized coordinates of the atoms of the basal surface clusters and water molecules.

When studying the adsorption of one $\mathrm{H}_{2} \mathrm{O}$ molecule on the siloxane and hydroxyl basal surfaces, the $\delta^{\mathrm{BSSE}}$ values were obtained equal to $-0.21 \mathrm{eV}$ and $-0.62 \mathrm{eV}$, respectively. Taking these corrections into account in Formula (5) leads to underestimated adsorption energies of $-0.15 \mathrm{eV}$ and $0.05 \mathrm{eV}$, respectively. In the $\mathrm{HF}$ approximation, the $\delta^{\mathrm{BSSE}}$ values turn out to be $-0.17 \mathrm{eV}$ and $-0.51 \mathrm{eV}$ which yields the adsorption energies of $-0.11 \mathrm{eV}$ and $0.31 \mathrm{eV}$, respectively. The reasons for the significant effect of BSSE on the results of calculating the energies were sufficiently discussed in the literature. In [24], counterpoise correction overcorrecting is associated with the use of small basis sets. In [25], this was demonstrated for the compounds with hydrogen bonds which include the cases of absorption of $\mathrm{H}_{2} \mathrm{O}$ molecules by the basal surfaces of kaolinite considered in this work. 


\subsection{Formation of the IR-Spectrum in the Range of 2500-4500 $\mathrm{cm}^{-1}$}

The IR spectrum of water molecules in a vaporous state has been studied for a long time. It is noted in [26] that the spectral range includes three modes: one stretching mode $\left(v_{1}\right)$ and two modes of deformation vibrations $\left(v_{2}, v_{3}\right)$. Still, only two modes with wavenumbers $1595 \mathrm{~cm}^{-1}\left(v_{2}\right)$ and $3755 \mathrm{~cm}^{-1}\left(v_{3}\right)$ are observed experimentally. These modes appear in the spectrum as rather intense bands, so that the band associated with the $v_{1}$ mode is masked by the overlapping intense $v_{3}$ band. The calculation of molecular constants performed in [26] made it possible to determine the bond angle and the $\mathrm{O}-\mathrm{H}$ distance. They amounted to $104^{\circ} 31^{\prime}$ and $0.9580 \AA$, respectively.

In [27], the changes in the IR spectrum were studied upon going from the vapor-water interface to water clusters and water in a liquid state. It is noted that the spectrum of the vapor-water interface in the range of $2800-3800 \mathrm{~cm}^{-1}$ is assigned to three vibrations with wavenumbers of about 3200,3400 , and $3700 \mathrm{~cm}^{-1}$. The spectrum contains two main features: a broad band in the range of wavenumbers from 3100 to $3500 \mathrm{~cm}^{-1}$ and a relatively sharp peak at $3690 \mathrm{~cm}^{-1}$ associated with the vibrations in free $\mathrm{OH}$ molecular bonds. In the liquid state of water, a sharp peak is not observed, since in the bulk of liquid water all free molecular bonds disappear due to intermolecular interaction. In this case, the resonances between 3100 and $3500 \mathrm{~cm}^{-1}$ are usually attributed to the stretching modes of hydrogen-bonded $\mathrm{OH}$.

In this study, we calculated fragments of the IR spectra of kaolinite of different moistures. The formation of the spectra is associated with the vibrations of oxygen and hydrogen atoms of the basal surfaces, and those of water molecules. In experimental studies, this spectral region is recorded in the range of $2500-4500 \mathrm{~cm}^{-1}$.

As can be seen from Figure $2 b$, the contribution of the surface to the IR spectrum is determined by the vibrations of $\mathrm{O}$ and $\mathrm{H}$ atoms forming free molecular bonds (Figure 7). The atoms of the siloxane surface of the mineral do not have spectral bands in the indicated range of wavenumbers (Figure 1).
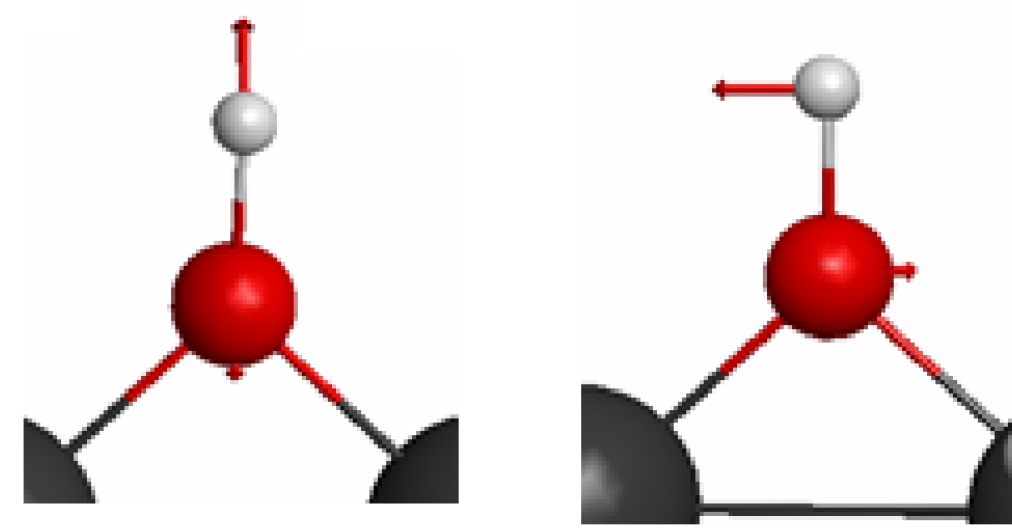

Figure 7. Vibrations in free bonds of $\mathrm{O}$ and $\mathrm{H}$ atoms on a dry hydroxyl surface. Red arrows indicate the direction of motion of atoms during vibrations.

The adsorption of water molecules by the basal surfaces of the mineral leads to the emergence of a chemical bond between the surface atoms and the atoms of water molecules, as a result of which the positions of the bands of kaolinite and water change, new collective vibrations of the surface and water atoms arise, as well as the collective vibrations of the atoms of several water molecules. These oscillations are illustrated in Figure 8. 


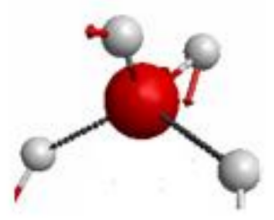

a

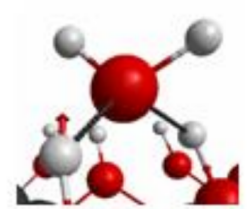

b

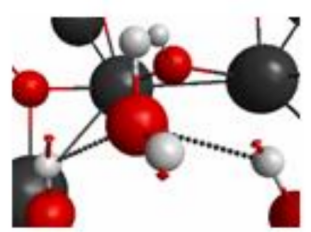

C

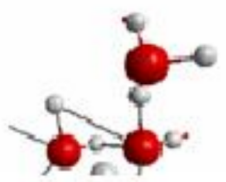

d

Figure 8. Fragments of the hydroxyl surface and adsorbed water molecules. Sown are the vibrations of the atoms of water molecule (a), of atoms of the surface of a mineral (b), collective vibrations of atoms of water and the surface of the mineral (c), and collective vibrations of atoms of different water molecules (d). Red arrows indicate the direction of motion of atoms during oscillations.

When calculating theoretical profiles of the bands, the width of the lines associated with free vibrations of surface ions was estimated from the experiment and taken equal to $5 \mathrm{~cm}^{-1}$. The line widths associated with the vibrations of water ions and collective vibrations are taken from [14]; they are $400-500 \mathrm{~cm}^{-1}$, depending on the moisture content. These line widths were used to generate Lorentzian curves representing individual calculated vibrations which were summed up. Calculated IR spectra of the hydroxyl and siloxane surfaces at different numbers of adsorbed water molecules are shown in Figures 9 and 10. In the spectra, the lines associated with the vibrations of hydrogen atoms closing chemical bonds broken during the construction of clusters are not considered. In addition, for comparison of theory and experiment, the theoretical spectra of the hydroxyl surface are shifted towards lower wavenumbers by $320 \mathrm{~cm}^{-1}$, and those of the siloxane surface by $600 \mathrm{~cm}^{-1}$. The reasons leading to the need for a shift in the theoretical spectra can be related to the limited size of the clusters, and the basis sets used to describe one-electron orbitals, as well as with the need to use a multiconfigurational approach when calculating the potentials of oscillators. Figures 9 and 10 also show the experimental $\mathrm{O}-\mathrm{H}$ vibration lines of the hydroxyl surface and water from [14].

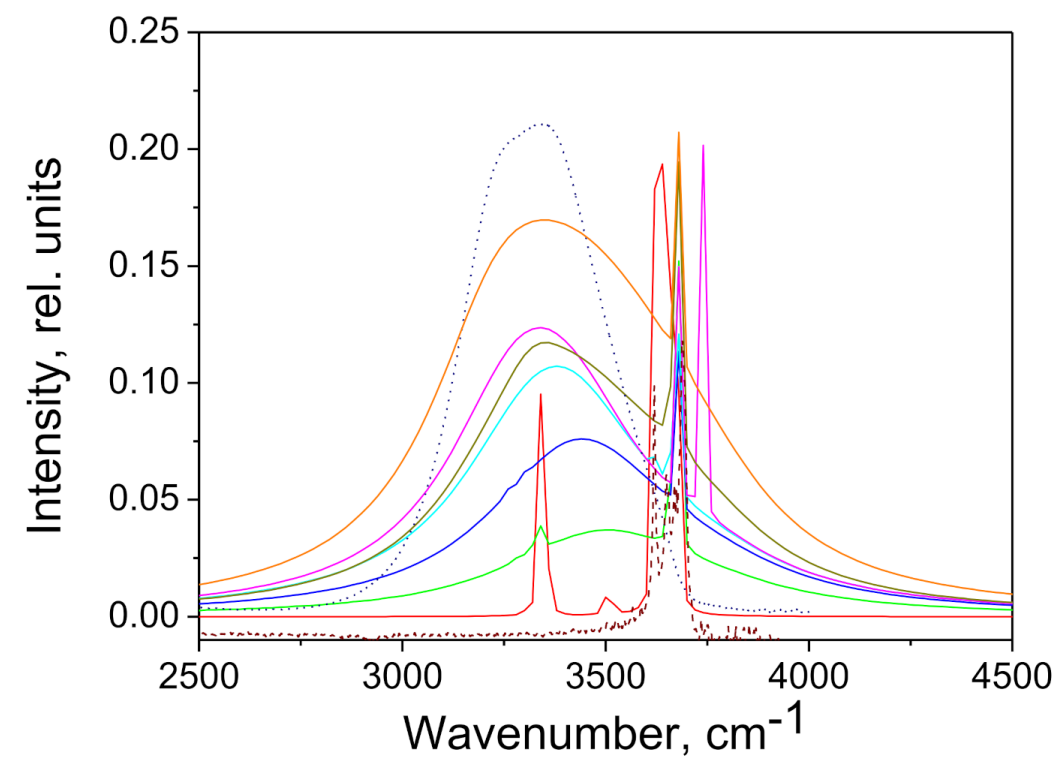

Figure 9. IR spectra formed by hydroxyl surface and water molecules. Number of adsorbed water molecules: 0—(red); 1—-(green); 2-(blue); 3-(cyan); 4-(magenta); 5-_(dark yellow); 6 - (orange). The bands of the hydroxyl surface and liquid water are shown by the dashed line (wine) and the dot line (navy), respectively. 


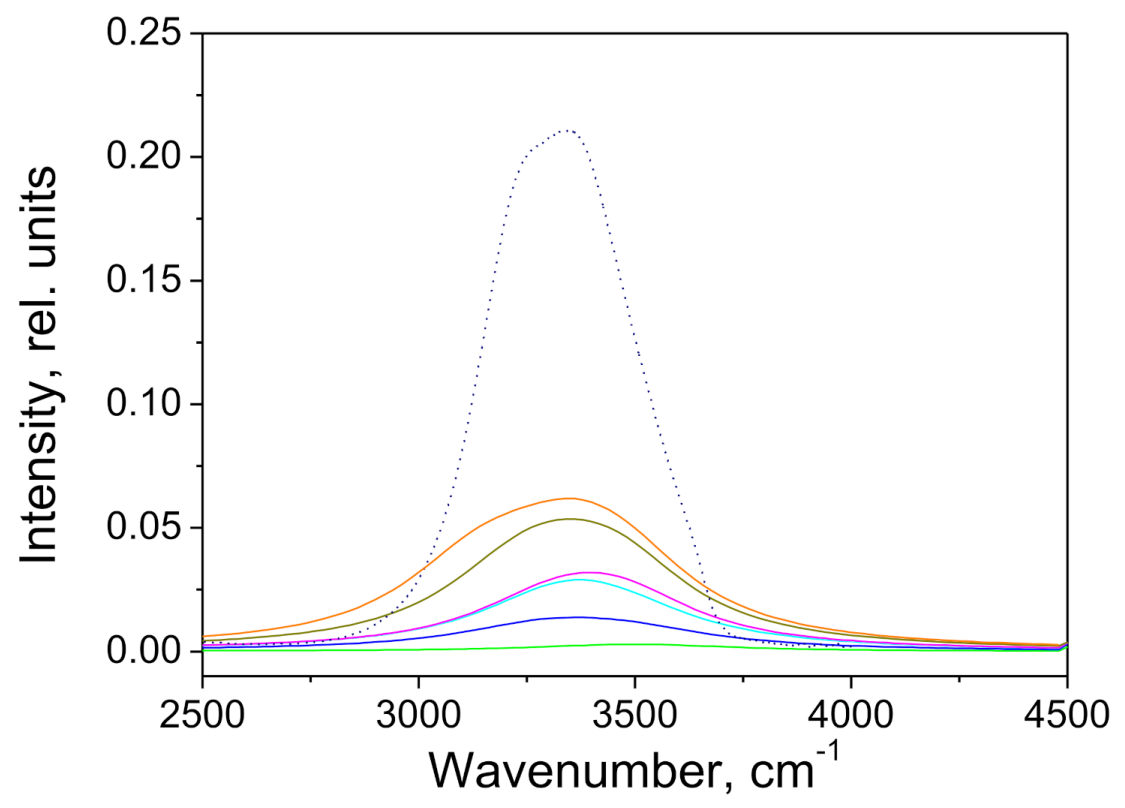

Figure 10. IR spectra formed by a siloxane surface and water molecules. Number of adsorbed water molecules: $1-$ (green); $2-$ (blue); 3-(cyan); $4-$ (magenta); 5-(dark yellow); 6(orange). Liquid water band is shown by the dot line (navy).

The profiles of theoretical IR spectra are compared with the experiment in Figure 11. Solid and dashed lines show the results for the hydroxyl and siloxane basal surfaces, respectively. The spectra in Figure 11 are normalized so that the intensities of theoretical and experimental bands are equal at $3330 \mathrm{~cm}^{-1}$. Relative intensities of the experimental bands were assumed to differ by a factor of 2.7 at $3360 \mathrm{~cm}^{-1}$ [14].

Theoretical spectra were calculated by taking into account five water molecules near the basal surfaces. According to Table 1, this case for a hydroxyl surface corresponds to a moisture content of $26.8 \%$. The experimental spectrum in Figure 11 was measured at a sample moisture content of $25 \%$. It is seen that the theory reproduces the main features of the experimental spectrum. The principal disagreement is in overestimated intensity of the theoretical spectrum above $3500 \mathrm{~cm}^{-1}$; this may be caused by the limitations of the theoretical model used.

The placement of five water molecules near the siloxane surface, according to Table 1, corresponds to a moisture of $16.3 \%$. The experimental spectrum shown in Figure 11 was measured at a sample moisture content of $18 \%$. It is seen that in the case of a siloxane surface, the theoretical spectrum does not contain resonance lines in the range of $3600-3750 \mathrm{~cm}^{-1}$; this is explained by the absence of the $\mathrm{O}-\mathrm{H}$ bonds on the siloxane surface.

The calculation results shown in Figures 9 and 10 are used to depict the dependence of the position of maximum in the wide band on the moisture content of the sample. This dependence is shown in Figure 12. When plotting the graphs in Figure 12, the relationship between the number of adsorbed water molecules and the moisture content of the sample given in Table 1 was used. Figure 12 shows that the theory reproduces experimentally found [14] optical effect of the shift of the wide band towards smaller wavenumbers with an increase in the moisture content of kaolinite. This effect can be interpreted as an increase in the oscillators mass that determine the features of the spectrum in the considered range of wavenumbers, due to additional chemical bond of surface atoms with the atoms of water molecules. This influence increases as the moisture content of the mineral rises. 


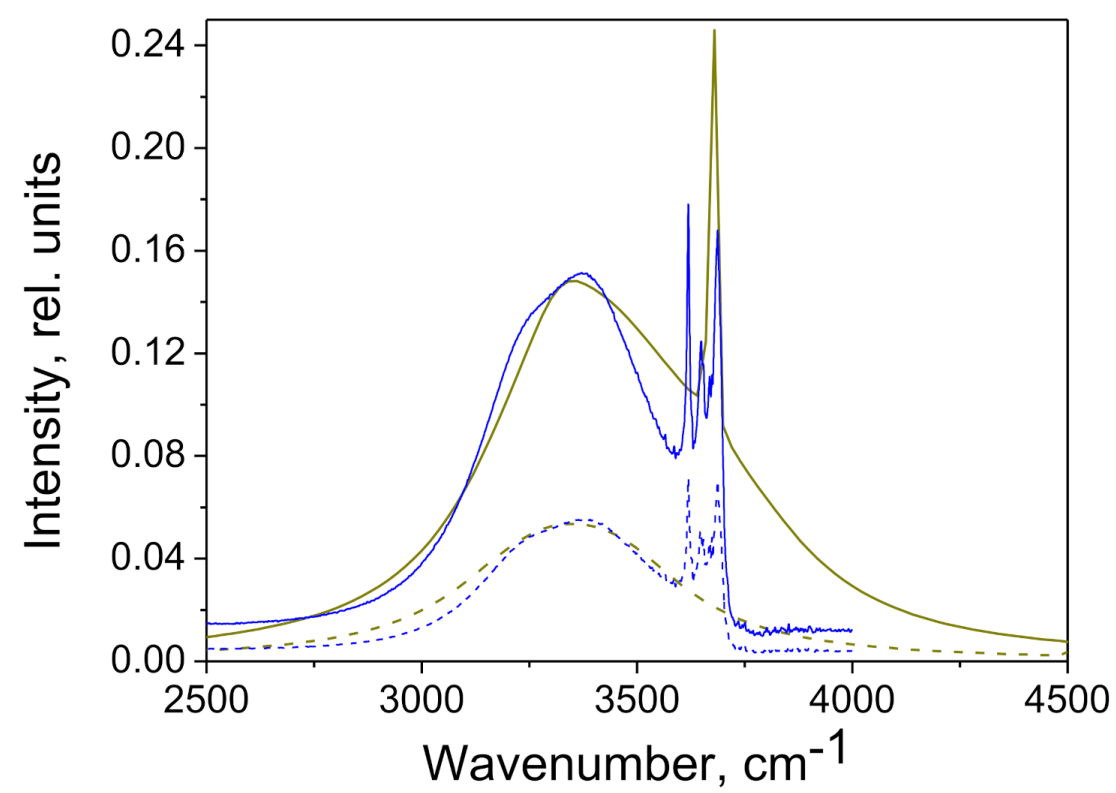

Figure 11. Comparison of experimental (blue lines) and theoretical (dark yellow lines), IR spectra of kaolinite formed by hydroxyl (solid lines), and siloxane (dashed lines) basal surfaces.

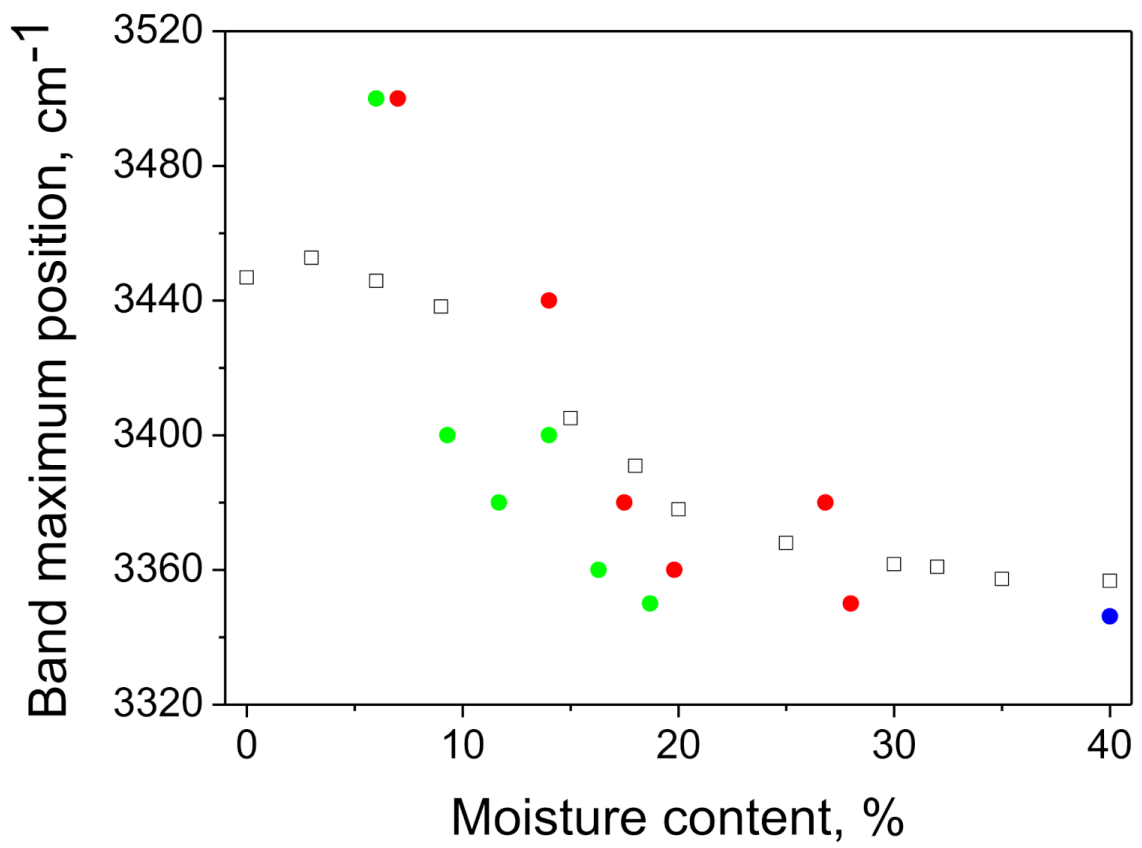

Figure 12. Spectral position of the wide band maximum (see Figures 9 and 10) depending on the moisture content of kaolinite. Experimental results [14]: •-liquid water, $\square-$ kaolinite of different moisture content; theoretical results of this work: •-hydroxyl surface; •-siloxane surface.

When analyzing the data shown in Figure 12, it is necessary to take into account the adsorption energy on the basal surfaces of the mineral. According to the data shown in Figure 6, hydration of the siloxane surface is energetically less favorable compared to the hydroxyl surface, at least up to moisture content at the plastic limit when water begins to fill the pores of the sample. At the same time, the reported results indicate that the nature of the dependence of the wavenumber on moisture is retained even in the case of hydration of the siloxane surface.

Figure 13 shows experimental and calculated relative intensities of the water band depending on the moisture content of kaolinite. It can be seen that the calculation repro- 
duces an experimentally discovered tendency to increase in the intensity of the band with an increase in the moisture content of the sample [14]. This can be explained by an increase in the number of oscillators with an increase in the moisture content of the mineral.

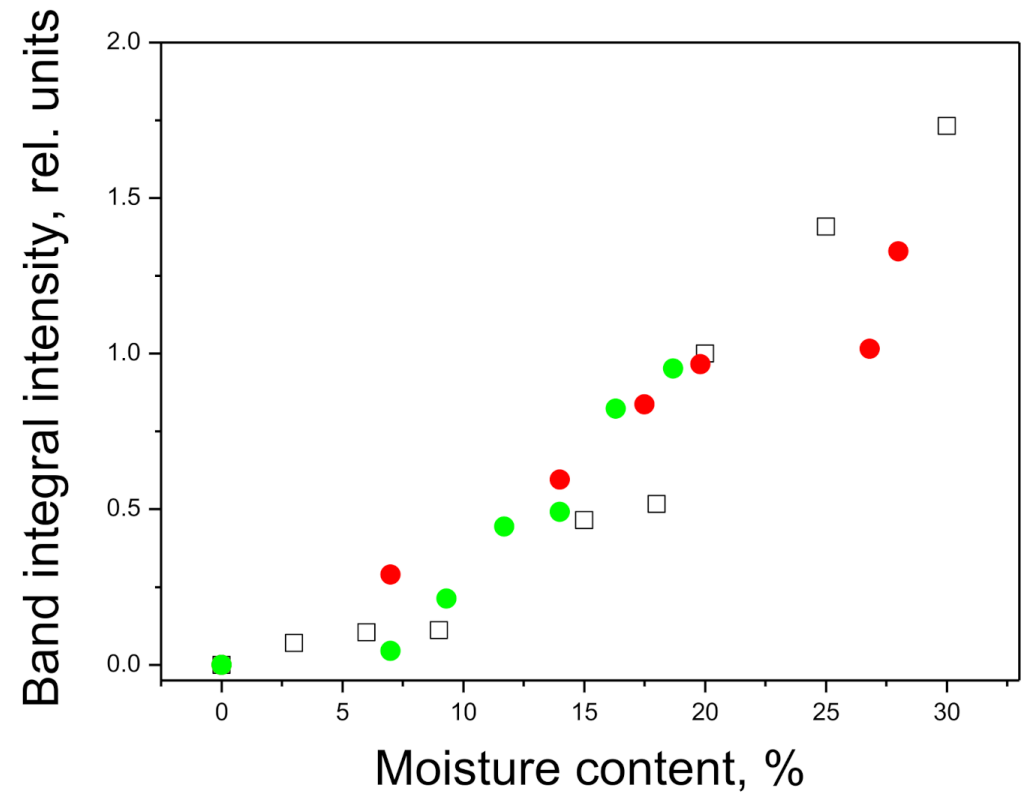

Figure 13. Integral intensity of the wide water band depending on the moisture content of kaolinite. Experiment [14]— $\square$; theoretical results of this work: •—hydroxyl surface; •—siloxane surface.

\section{Conclusions}

This paper presents theoretical study of the effect of hydration on the formation of the features of the IR spectrum of kaolinite in the range of $2500-4500 \mathrm{~cm}^{-1}$. Theoretical calculations of the electronic and spatial structure of hydrated kaolinite are performed in the Hartree-Fock approximation. The calculation results were used to simulate the IR spectra at different moisture content of the mineral. When calculating the adsorption energies, fourth-order electronic correlations were taken into account within the MøllerPlesset perturbation theory. Calculated adsorption energies are in good agreement with the values obtained by other methods which indicates the adequacy of the approximations used in this work.

Simulation of the profile of theoretical IR spectrum in the range of $2500-4500 \mathrm{~cm}^{-1}$ made it possible to qualitatively interpret experimentally discovered regularities, i.e. the shift of the maximum of the band towards smaller values of the wavenumber and the increase in the relative intensity of the band with an increase in the moisture content of kaolinite samples. This effect is interpreted as a manifestation of the chemical bond between the surface atoms of kaolinite and the atoms of water molecules. This interaction leads to an increase in the oscillators' masses and in their number with an increase in the moisture content of the mineral.

Author Contributions: Conceptualization, V.Y. and A.K.; methodology, V.Y.; investigation, T.N., Y.E. and A.M.; writing - original draft preparation, V.Y.; writing-review and editing, A.K.; visualization, T.N., Y.E. and A.M.; supervision, V.Y.; project administration, V.Y.; All authors have read and agreed to the published version of the manuscript.

Funding: This work was supported by the Russian Science Foundation (Grant No. 21-79-20005).

Conflicts of Interest: The authors declare no conflict of interest. 


\section{References}

1. Andrade, F.A.; Al-Qureshi, H.A.; Hotza, D. Measuring the plasticity of clays: A review. Appl. Clay Sci. 2011, 51, 1-7. [CrossRef]

2. Tiwari, B.; Ajmera, B. A new correlation relating the shear strength of reconstituted soil to the proportions of clay minerals and plasticity characteristics. Appl. Clay Sci. 2011, 53, 48-57. [CrossRef]

3. Zhao, H.; Ge, L.; Petry, T.M.; Sun, Y.-Z. Effects of chemical stabilizers on an expansive clay. KSCE J. Civ. Eng. 2014, 18, 1009-1017. [CrossRef]

4. de Oliveira Modesto, C.; Bernardin, A.M. Determination of clay plasticity: Indentation method versus Pfefferkorn method. Appl. Clay Sci. 2008, 40, 15-19. [CrossRef]

5. Hatch, C.D.; Wiese, J.S.; Crane, C.C.; Harris, K.J.; Kloss, H.G.; Baltrusaitis, J. Water adsorption on clay minerals as a function of relative humidity: Application of BET and Freundlich adsorption models. Langmuir. 2012, 28, 1790-1803. [CrossRef]

6. Vasilyeva, M.A.; Gusev, Y.A.; Shtyrlin, V.G.; Greenbaum Gutina, A.; Puzenko, A.; Ishai, P.B.; Feldman, Y. Dielectric relaxation of water in clay minerals. Clays Clay Miner. 2011, 62, 62-73. [CrossRef]

7. Kaufhold, S.; Hein, M.; Dohrmann, R.; Ufer, K. Quantification of the mineralogical composition of clays using FTIR spectroscopy. Vib. Spectrosc. 2012, 59, 29-39. [CrossRef]

8. Morozov, A.; Vasilchenko, A.; Kasprzhitskii, A.; Lazorenko, G.; Yavna, V.; Kochur, A. Express method for determining the composition of soils based on IR spectroscopy technology. Vib. Spectrosc. 2021, 114, 103258. [CrossRef]

9. Waruru, B.K.; Shepherd, K.D.; Ndegwa, G.M.; Kamoni, P.T.; Sila, A.M. Rapid estimation of soil engineering properties using diffuse reflectance near infrared spectroscopy. Biosyst. Eng. 2014, 121, 177-185. [CrossRef]

10. Kasprzhitskii, A.; Lazorenko, G.; Yavna, V.; Daniel, P. DFT theoretical and FT-IR spectroscopic investigations of the plasticity of clay minerals dispersions. J. Mol. Struct. 2016, 1109, 97-105. [CrossRef]

11. Hu, X.L.; Michaelides, A. Water on the hydroxylated (001) surface of kaolinite: From monomer adsorption to a flat 2D wetting layer. Surf. Sci. 2008, 602, 960-974. [CrossRef]

12. Chen, J.; Min, F.-F.; Liu, L.-Y.; Liu, C.-F. Mechanism research on surface hydration of kaolinite, insights from DFT and MD simulations. Appl. Surf. Sci. 2019, 476, 6-15. [CrossRef]

13. Šolc, R.; Gerzabek, M.H.; Lischka, H.; Tunega, D. Wettability of kaolinite (001) surfaces - Molecular dynamic study. Geoderma. 2011, 169, 47-54. [CrossRef]

14. Nazdracheva, T.F.; Kukharskii, A.V.; Kasprzhitskii, A.S.; Lazorenko, G.I.; Yavna, V.A.; Kochur, A.G. Study of the Features of the Formation of Water Films on the Surfaces of Montmorillonite and Kaolinite by Infrared Spectroscopy. Opt. Spectrosc. 2021, 129, 270-275. [CrossRef]

15. Liu, X.; Lu, X.; Wang, R.; Meijer, E.J.; Zhou, H.; He, H. Atomic scale structures of interfaces between kaolinite edges and water. Geochim. Cosmochim. Acta. 2012, 92, 233-242. [CrossRef]

16. Bish, D.L. Rietveld refinement of the kaolinite structure at 1.5 K. Clays Clay Miner. 1993, 41, 738-744. [CrossRef]

17. Bode, B.M.; Gordon, M.S. MacMolPlt: A graphical user interface for GAMESS. J. Mol. Graph. Model. 1998, 16, 133-138. [CrossRef]

18. Firefly Version 8. Available online: http://classic.chem.msu.su/gran/firefly/index.html (accessed on 22 August 2021).

19. Schmidt, M.W.; Baldridge, K.K.; Boatz, J.A.; Elbert, S.T.; Gordon, M.S.; Jensen, J.H.; Koseki, S.; Matsunaga, N.; Nguyen, K.A.; Su, S.; et al. General atomic and molecular electronic structure system. J. Comput. Chem. 1993, 14, 1347-1363. [CrossRef]

20. Boys, S.F. and Bernardi, F. The calculation of small molecular interactions by the differences of separate total energies. Some procedures with reduced errors. Molecular Physics. 1970, 19, 553-556. [CrossRef]

21. Kruglikov, A.; Vasilchenko, A.; Kasprzhitskii, A.; Lazorenko, G. Atomic-level understanding of interface interactions in a halloysite nanotubes-PLA nanocomposite. RSC Adv. 2019, 9, 39505-39514. [CrossRef]

22. Kasprzhitskii, A.S.; Lazorenko, G.I.; Yavna, V.A.; Kochur, A.G. A study of the structural and spectral characteristics of free and bound water in kaolinite. Opt. Spectrosc. 2016, 121, 357-363. [CrossRef]

23. Dolinar, B. Predicting the hydraulic conductivity of saturated clays using plasticity-value correlations. Appl. Clay Sci. 2009, 45, 90-94. [CrossRef]

24. Liedl, K.R. Dangers of counterpoise corrected hypersurfaces. Advantages of basis set superposition improvement. J. Chem. Phys. 1998. 108, 3199-3204. [CrossRef]

25. Halkier, A.; Klopper, W.; Helgaker, T.; Jørgensen, P.; Taylor, P.R. Basis set convergence of the interaction energy of hydrogenbonded complexes. J. Chem. Phys. 1999, 111, 9157-9167. [CrossRef]

26. Darling, B.T.; Dennison, D.M. The Water Vapor Molecule. Phys. Rev. 1940, 57, 128-139. [CrossRef]

27. Du, Q.; Superfine, R.; Freysz, E.; Shen, Y.R. Vibrational Spectroscopy of Water at the Vapor/Water Interface. Phys. Rev. Lett. 1993, 70, 2313-2316. [CrossRef] [PubMed] 